\title{
A Solution to the World Economic and Financial Crisis
}

\section{Jiancheng Liu}

Dongfang Boiler Co., Ltd., Zigong, China

Email: decljc@163.com

Received 16 April 2016; accepted 15 May 2016; published 23 May 2016

Copyright (C) 2016 by author and OALib.

This work is licensed under the Creative Commons Attribution International License (CC BY). http://creativecommons.org/licenses/by/4.0/
(c) (i)
Open Access

\section{Abstract}

This paper introduces the matter growth equation, discusses the patterns of economic growth and the growth of labor productivity, analyses the causes of the stagnation and economic crisis and the relationship between the value of currency and the quality of products, and then puts forward the currencies reform and the income distribution schemes, and provides a solution for the world economic and financial crisis. JEL Codes: 043, J31, K20.

\section{Keywords}

\section{Economic Growth Patterns, Economic Crisis, Currency Reform, Income Distribution}

\section{Subject Areas: Economics, Political Economy}

\section{1. 事物成长方程}

宇宙万物一旦出生, 都应随时间一起成长, 所以有: $\frac{\mathrm{d} q}{q}=\lambda \frac{\mathrm{d} t}{t}$, 式中 $q$ 为宇宙万物(包括人类)的规 模(大小)和质量(品质), $t$ 为时间, $\lambda$ 为系数。引入事物势(记为 $P$ )的概念, 它被定义为某一事物的数量 $(Q)$ 与该事物的质量水平 $(q)$ 的乘积, 那么有: $P=Q \cdot q, \frac{\mathrm{d} P}{P}=\frac{\mathrm{d} Q}{Q}+\frac{\mathrm{d} q}{q}$, 即事物势的增长率 $\frac{\mathrm{d} P}{P}$ 等于事物数 量的增长率 $\frac{\mathrm{d} Q}{Q}$ 和该事物质量水平的增长率 $\frac{\mathrm{d} q}{q}$ 之和。由于事物成长方程的特性, 随着时间的推移, 其成 长(发展)速度呈现先快后慢的属性, 当时间无限长时, 发展速度趋近于 0 [1]。

\section{2. 经济增长方式}

从微观层面看, 全社会产出(即国民收入)的名义值 $y^{\prime}$ 的大小主要取决于全社会产品总产量的大小 $(Q)$ 
和产品平均质量水平 $(q)$ 及产品平均价格水平 $\left(P_{0}\right)$ 的高低。因此, 全社会产出(国民收入)的名义值等于全社 会产品总产量与产品平均质量水平和产品平均价格水平三者的积: $y^{\prime}=Q \cdot q \cdot P_{0}, \frac{\mathrm{d} y^{\prime}}{y^{\prime}}=\frac{\mathrm{d} Q}{Q}+\frac{\mathrm{d} q}{q}+\frac{\mathrm{d} P_{0}}{P_{0}}$, 式中, $\frac{\mathrm{d} Q}{Q}$ 为产品产量的增长率, $\frac{\mathrm{d} q}{q}$ 为产品平均质量水平增长率, $\frac{\mathrm{d} P_{0}}{P_{0}}$ 为产品平均价格水平增长率(即通 货膨胀率), 国民收入名义增长率 $\frac{\mathrm{d} y^{\prime}}{y^{\prime}}$ 等于这三者之和。而 $\frac{\mathrm{d} y}{y}=\frac{\mathrm{d} y^{\prime}}{y^{\prime}}-\frac{\mathrm{d} P_{0}}{P_{0}}=\frac{\mathrm{d} Q}{Q}+\frac{\mathrm{d} q}{q}$, 即国民收入增长率(实 际值) $\frac{\mathrm{d} y}{y}$ 等于产品产量增长率 $\frac{\mathrm{d} Q}{Q}$ 和产品平均质量水平增长率 $\frac{\mathrm{d} q}{q}$ 之和。提高产品平均质量水平, 会成正 比地增加国民收入，大大促进经济增长 [2]。

实际上, 我们可以称产品产量增长率为经济增长率的外延部分, 而称产品平均质量水平增长率为经 济增长率的内涵部分; 通过扩大产量获得的增长为外延式增长, 通过提高质量水平获得的增长为内涵式 增长, 两种方式皆用而获得的增长为复合式增长。外延式增长是不可能长期持续的, 当产品饱和或过剩 时, 其增长率为零或为负。相当长时间以来及当前, 我国采用的是通过扩大产量获得增长的外延式增长 方式, 我们必须将这种增长方式转变为通过提高质量水平获得增长的内涵式增长方式, 即在提高产品质 量上下功夫。这是我国目前和未来急需要解决的问题[2]。

\section{3. 劳动生产率的增长}

$$
\frac{\mathrm{d}(y / L)}{y / L}=\frac{\mathrm{d} q}{q}+\frac{\mathrm{d} Q}{Q}-\frac{\mathrm{d} L}{L}=\frac{\mathrm{d} q}{q}+\frac{\mathrm{d}(Q / L)}{Q / L} \text {, 即劳动生产率的增长率等于质量水平增长率与人均产品产量 }
$$
$\frac{Q}{L}$ 的增长率之和。所以, 要提高劳动生产率, 就必须不断提高产品质量水平和人均产品产量。

同样, 我们可以称人均产品产量增长率为劳动生产率增长率的外延部分, 而称产品平均质量水平增 长率为劳动生产率增长率的内涵部分; 通过提高人均产品产量获得的增长为外延式增长, 通过提高质量 水平获得的增长为内涵式增长, 两种方式皆用而获得的增长为复合式增长。外延式增长是不可能长期持 续的, 当人均产品产量达到极限时, 其增长率为零或为负。相当长时间以来及当前, 我国采用的是通过 提高人均产品产量获得增长的外延式增长方式, 我们必须将这种增长方式转变为通过提高产品平均质量 水平获得增长的内涵式增长方式, 亦即在提高产品质量上下功夫。这也是我国目前和未来急需要解决的 问题[2]。

\section{4. 产生滞胀和经济危机的原因}

当产品需求趋于饱和时, 正常需要的产品产量增长率 $\frac{\mathrm{d} Q}{Q}=0$, 供需平衡, 产品产量不能再继续增长 了, 滞涨便产生了; 如果产品产量再增长, $\frac{\mathrm{d} Q}{Q}>0$, 那必然会出现生产过剩, 产品积压, 不能实现销售, 马克思所说的生产过剩的经济危机便产生了。这时, 产品产量的增长对经济增长的贡献为 0 , 如果产品 平均质量水平不增长反而下降, 即产品平均质量水平增长率 $\frac{\mathrm{d} q}{q} \leq 0$ 时, 那么国民收入的增长率也将小于 0 , 即 $\frac{\mathrm{d} y}{y} \leq 0$, 经济便出现衰退。为了化解危机, 必须减产, 使产品产量增长率 $\frac{\mathrm{d} Q}{Q}<0$; 同时, 要提高 产品平均质量水平增长率, 使 $\frac{\mathrm{d} q}{q}>0$, 而且使产品平均质量水平增长率的绝对值 $\left|\frac{\mathrm{d} q}{q}\right|$ 大于产品产量增长 
率的绝对值 $\left|\frac{\mathrm{d} Q}{Q}\right|$ (即减产率), 那么, 国民收入便会继续增长, 其增长率 $\frac{\mathrm{d} y}{y}=\left|\frac{\mathrm{d} q}{q}\right|-\left|\frac{\mathrm{d} Q}{Q}\right|>0$ 。这样一来, 经济危机便被克服了。

所以, 要克服世界目前的经济危机, 必须先减产(压缩产能), 同时提高产品平均质量水平, 并且使产 品平均质量水平增长率的绝对值大于产品产量增长率的绝对值(即减产率)。经济发展的质量由产品的平均 质量水平决定, 对于我国经济发展来说, 我们只能采取领先发展的策略, 即在提高产品质量、开发新产 品和培养新产业等方面下功夫, 提高经济发展的速度和质量。

\section{5. 币值与产品质量}

从理论上讲, 货币最小单位的价值代表了最基本(价格最低)产品的价值, 所以, 最基本产品的质量水 平与货币最小单位的价值成正比, 哪一个国家或地区货币最小单位的价值越高, 则其产品平均质量水平 越高。从实际情况看, 币值较高的发达国家或地区, 其产品质量水平的确更高。例如, 英国、美国、德 国和以前的日本, 他们的产品质量确实高人一筹。当下, 日元大幅贬值, 表明日本产品的质量也在大幅 下降。

对中国而言, 解放前一个现大洋面值为一元, 而现在要卖到 100 多元人民币。也就是说, 中国货币 的价值从 1949 年到现在贬值了 100 多倍, 解放前的一个小钱就能相当于现在的 1 元人民币。当然, 现在 最便宜的单件产品能卖到 0.1 元人民币左右。所以, 可以认为, 现在的产品平均质量水平只相当于 1949 年前的 $1 / 10$, 中国产品的质量水平是逐年退步的。

要使现在的平均产品质量水平与 1949 年前相当, 就有必要将现在的人民币贬值 10 倍。为了给平均 产品质量水平的提高(经济增长)留有余地, 比如说, 到 2049 年建国 100 周年时, 我国的产品平均质量水 平较 1949 年前的水平提高 10 倍, 则有必要将现行的人民币贬值 100 倍。所以, 我国可以发行新的人民 币, 其面值为 1 分至 10 元不等, 同时废除现行人民币, 并规定现行人民币以 100 元对 1 元的比例与新人 民币兑换。同时, 通过国际磋商说服世界其它国家和地区采取相同的政策。这样一来, 当下的世界经济 和金融危机便迎刃而解。

\section{6. 对人力资源的考虑}

目前, 人力资源仅被当作与其它三种生产要素(资本、技术、管理)对等的要素, 人力资源成本(工资 等)作为管理成本之一计入财务考虑。且从我国的情况看, 工资收入在国民收入中所占的比重很低, 仅 35\% 左右。实际上, 人力资源不仅是不同于实物资本的独立要素, 同时也是一种特殊资本, 其对经济增长的 贡献远大于实物资本。所以在投资中, 不仅包括实物资本额, 而且应当包括人力资本额。那么, 人力资 本怎样作价呢? 对中国而言, 可根据个人受教育程度、工作能力和对企业的贡献不同进行作价, 将人力 资本根据自己在整个企业投资额(总的实物资本额 + 总的人力资本额)中所占的比例参与企业利润的等 权分配。所以，人力资源除得到工资外，还以资本的形式参与企业利润的分配。

在宏观经济模型中，投资额 I 也应等于实物资本额 + 人力资本额的总和。即为保持国民经济增长， 多投入劳动和多投入实物资本, 具有同等的效用。于是, 员工和出资人会共同关心企业的利润, 不仅劳 动和资本的矛盾解决了, 充分就业也是比较容易实现的。

拿我所在的东方锅炉来说, 最近十多年来, 职工平均年工资收入为 $5 \sim 10$ 万元现行人民币(500 1000 元新人民币)不等, 考虑到职工的年龄和工龄结构, 假设平均工龄为 10 年, 那么东方锅炉职工的平均工 资总收入为 50 100 万元现行人民币(5000 10,000 元新人民币)不等。所以, 不妨将东方锅炉职工平均每 人按 80 万元现人民币 $(8000$ 元新人民币)作价。东方锅炉的实物资本净资产为 50 亿元现人民币, 其当年 
(2014 年)的净利润为 8.5 亿元现人民币, 而其员工总数为 4000 人, 平均每人按 80 万元现人民币作价, 即 企业总人力资本额为 32 亿元现人民币, 那么企业员工应分得总的利润份额 $\left(R_{h}\right)$ 如下:

$$
R_{h}=\frac{32}{50+32} \times 8.5=3.32 \text { 亿元现人民币. }
$$

\section{7. 结论}

要克服世界目前的经济危机, 必须先减产(压缩产能), 同时提高产品平均质量水平, 并且使产品平均 质量水平增长率的绝对值大于产品产量增长率的绝对值(即减产率)。经济发展的质量由产品的平均质量水 平决定, 对于我国经济发展来说, 我们只能采取领先发展的策略, 即在提高产品质量、开发新产品和培 养新产业等方面下功夫, 提高经济发展的速度和质量。

从理论上讲, 货币最小单位的价值代表了最基本(价格最低)产品的价值, 所以, 最基本产品的质量水 平与货币最小单位的价值成正比, 哪一个国家或地区货币最小单位的价值越高, 则其产品平均质量水平 越高。我国可以发行新的人民币, 其面值为 1 分至 10 元不等, 同时废除现行人民币, 并规定现行人民币 以 100 元对 1 元的比例与新人民币兑换。同时, 通过国际碰商说服世界其它国家和地区采取相同的政策。 这样一来，当下的世界经济和金融危机便迎刃而解。

人力资源除得到工资外, 还以资本的形式参与企业利润的分配。人力资本根据自己在整个企业投资 额(总的实物资本额 + 总的人力资本额)中所占的比例参与企业利润的等权分配。

保持国民经济增长, 多投入劳动和多投入实物资本, 具有同等的效用。员工和出资人会共同关心企 业的利润，不仅劳动和资本的矛盾解决了，充分就业也是比较容易实现的。

\section{参考文献 (References)}

[1] Liu, J. (2016) General Theory of Relativity for Development Economics. Open Access Library Journal, 3, e2535. http://dx.doi.org/10.4236/oalib.1102535

[2] Liu, J. (2016) Planned Macroeconomics. Open Access Library Journal, 3, e2561. http://dx.doi.org/10.4236/oalib.1102561 


\section{附录：事物成长方程的来源及应用}

\section{A.1. 事物成长方程的推导}

宇宙万物一旦出生, 都应随时间一起成长, 所以有:

$$
\frac{\mathrm{d} q}{q}=\lambda \frac{\mathrm{d} t}{t}
$$

式中 $q$ 为宇宙万物(包括人类)的规模(大小)和质量(品质), $t$ 为时间, $\lambda$ 为系数。由于对任何事物都可 以仅用质量(品质)和规模(大小)两个参数加以描述, 所以, 如果设某种事物诞生时的质量(品质)或规模(大 小)为 1 , 孕育时间为 $t_{0}$, 那么, 对(1)式积分, 有:

$$
\int_{1}^{q} \frac{\mathrm{d} q}{q}=\int_{t_{0}}^{t} \lambda \frac{\mathrm{d} t}{t}
$$

由此得到事物质量(品质)或规模(大小)成长方程如下:

$$
\ln q=\lambda\left(\ln t-\ln t_{0}\right)
$$

如果 $\lambda=1$

$$
\ln q=\ln t-\ln t_{0}
$$

表明事物质量(品质)或规模(大小)发展与时间同步, 称为同步发展。

如果 $0<\lambda<1$

$$
\ln q<\ln t-\ln t_{0}
$$

表明事物质量(品质)或规模(大小)发展落后于时间的步伐，称为滞后发展。

如果 $\lambda>1$

$$
\ln q>\ln t-\ln t_{0}
$$

表明事物质量(品质)或规模(大小)发展领先于时间, 称为领先发展。

如果 $\lambda<0$, 事物质量(品质)或规模(大小)将会出现衰退。

引入事物势 (记为 $P$ ) 的概念, 它被定义为某一事物的数量 $(Q)$ 与该事物的质量水平 $(q)$ 的乘积, 那么有:

$$
\begin{gathered}
P=Q \cdot q \\
\frac{\mathrm{d} P}{P}=\frac{\mathrm{d} Q}{Q}+\frac{\mathrm{d} q}{q}
\end{gathered}
$$

即事物势的增长率 $\frac{\mathrm{d} P}{P}$ 等于事物数量的增长率 $\frac{\mathrm{d} Q}{Q}$ 和该事物质量水平的增长率 $\frac{\mathrm{d} q}{q}$ 之和。

\section{A.2. 事物成长方程的应用一一中国经济的发展速度}

\section{A.2.1. 对过去数据的分析}

由于

$$
\frac{\mathrm{d} y}{y}=\frac{\mathrm{d} Q}{Q}+\frac{\mathrm{d} q}{q}
$$

式中, $y=$ 剔除了价格变动的实际产出或收入

$Q=$ 产品产量

$q=$ 产品平均质量水平 
即国民收入增长率(实际值) $\frac{\mathrm{d} y}{y}$ 等于产品产量增长率 $\frac{\mathrm{d} Q}{Q}$ 和产品平均质量水平增长率 $\frac{\mathrm{d} q}{q}$ 之和。

\section{1) 孕育期从文革结束开始算起}

假设从 1978 年底开始, 中国经济发展才走上正轨，并与时间同步发展。设发展前的孕育期为 1 年, 那么由公式(3), 有

$$
\begin{gathered}
\ln y=\ln t-\ln t_{0}=\ln 37-\ln 1=3.61 \\
y=\mathrm{e}^{3.61}=37 \text { 倍。 }
\end{gathered}
$$

而实际上, 到 2015 年底这 37 年中, 我国的实际 GDP(由于 GDP 与 GNI 相差不大, 可认为 $y=$ GNI = GDP)。只增长为 1978 年的 31.55 倍, 似乎没有做到同步发展, 更不用说领先发展了, 而只是一种滞后发 展。

如果将 1976 年 10 月粉碎 “四人帮” 至 1978 年底十一届三中全会召开之间的两年零两个月(2.167 年) 作为孕育期, 则同步发展的 $q$ 为:

$$
\begin{gathered}
\ln y=\ln t-\ln t_{0}=\ln 39.167-\ln 2.167=2.895 \\
y=\mathrm{e}^{2.895}=18.06 \text { 倍 }<31.55 \text { 倍 }
\end{gathered}
$$

这样一来, 便可以认为我国经济发展为领先发展。那么当 $y=31.55$ 倍时, 相当于同步发展到了哪一 年了?

$$
\ln t=\ln y+\ln t_{0}=\ln 31.55+\ln 2.167=4.225
$$

$t=\mathrm{e}^{4.225}=68.37$ 年, 即发展程度提前了 31.4 年左右, 已发展到 2046 年。我国经济实际发展速度是同 步发展速度的 $\frac{68.37}{37}=1.848$ 倍。

那么从 1977 年至 2015 年, 我国的产品产量发展速度如何呢?

以粮食生产为例, 1977 年我国粮食总产量为 3000 亿公斤, 而 2015 年为 6214 亿公斤, 即增长了 $107.1 \%$, 年均增长 $1.98 \%$ 。

由于钢铁、水泥、煤炭、电力等在 37 年间增加了较多, 不妨认为这 37 年间我国产品产量增加了 7 倍(准确数据应国家统计局提供), 即年均增加 5.4\%。那么, 可以计算出我国 2015 年的产品平均质量水平 提高为 1977 年的 4.507 倍, 即年均提高 $4.15 \%$ 。我国产品平均质量的发展速度如何呢?

同前, 仍假设从 1978 年底开始, 中国经济发展才走上正轨, 产品质量水平与时间同步发展。设发展 前的孕育期仍为两年零两个月(2.167 年), 那么由公式(3), 有

$$
\begin{gathered}
\ln q=\ln t-\ln t_{0}=\ln 39.167-\ln 2.167=2.895 \\
q=\mathrm{e}^{2.895}=18.06 \text { 倍。 }
\end{gathered}
$$

即年均提高 $8.1 \%$ 。

而实际为年均提高 $4.15 \%$, 该发展速度仅为同步发展速度的一半。那么, 如果我国产品平均质量水 平为同步发展, 目前的水平相当于发展到哪一年了呢?

由公式(3)，我们有

$$
\begin{aligned}
\ln t=\ln q+\ln t_{0} & =\ln 4.507+\ln 2.167=2.279 \\
t & =\mathrm{e}^{2.279}=9.77
\end{aligned}
$$

即目前的实际产品平均质量水平只相当于发展到了 1986 年。 


\section{2) 孕育期从中华人民共和国成立开始算起}

假设从 1949 年 10 月 1 日中华人民共和国成立之日起, 我国经济开始乃育, 而直到 1978 年底开始, 中国经济发展才走上正轨, 并与时间同步发展。那么我国经济的孕育期为 29 年, 那么由公式(3), 有

$$
\begin{gathered}
\ln y=\ln t-\ln t_{0}=\ln 67-\ln 29=0.837 \\
y=\mathrm{e}^{0.837}=2.31 \text { 倍。 }
\end{gathered}
$$

即年均增长 $2.29 \%$ 。

而实际上, 到 2015 年底这 37 年中, 我国的实际 GDP 增长为 1978 年的 31.55 倍, 实现了大幅度的 领先发展。

那么从 1977 年至 2015 年, 我国的产品产量发展速度如何呢?

以粮食生产为例, 1977 年我国粮食总产量为 3000 亿公斤, 而 2015 年为 6214 亿公斤, 即增长了 $107.1 \%$, 年均增长 $1.98 \%$ ，没能实现同发展，而是一种滞后发展。

但是, 由于钢铁、水泥、煤炭、电力等在 37 年间增加了较多, 不妨认为这 37 年间我国产品产量增 加了 7 倍(准确数据应国家统计局提供), 即年均增加 5.4\%, 也实现了大幅度的领先发展。同时, 可以计 算出我国 2015 年的产品平均质量水平提高为 1977 年的 4.507 倍, 即年均提高 $4.15 \%$, 我国产品平均质量 水平同样实现了较大幅度的领先发展。

对上述分析进行比较, 可以发现, 事物(包括一个国家的经济水平)的孕育期越长, 其出生后的发展速 度越慢; 孕育期越短, 其出生后的发展速度越快。在事物出生以后, 其初期发展(成长)速度较快, 而随着 时间的推移, 其发展(成长)速度会越来越慢, 最后将趋近于 0 。即新生事物发展(成长)快, 旧事物发展(成 长)慢, 所以要鼓励新生事物的诞生和发展, 并对旧事物进行改造, 促其新生。改革开放即是促进了国家 的新生。对经济水平而言, 新兴国家的发展速度将会明显高于发达国家。

\section{A.2.2. 对 2016 年数据的要求}

假设我国经济仍保持这 37 年来的平均发展速度，那么有

$$
\frac{\mathrm{d} y}{y_{2015}}=\frac{\mathrm{d} Q}{\mathrm{~d} Q_{2015}}+\frac{\mathrm{d} q}{\mathrm{~d} q_{2015}}=5.4 \%+4.15 \%=9.55 \% .
$$

即只要 2016 年我国 GDP 实际增长 9.55\%, 就延续了这 37 年来的良好发展趋势。但是, 由于国际经 济、金融危机的影响，我国经济的发展速度应适当调低，比如 7\%。同时，由于国际经济危机对我国产品 出口的严重影响, 我们应适当调低产品产量的增速, 比如从 $5.4 \%$ 调低为 $2 \%$, 而适当调高产品平均质量 水平的提高率，比如从 $4.15 \%$ 调高为 $6 \%$, 从而保证实际 GDP7-8\%的增速。

\section{A.2.3. 今后的发展趋势}

由于事物成长方程的特性, 随着时间的推移, 其成长(发展)速度呈现先快后慢的属性, 当时间无限长 时, 发展速度趋近于 0 。对于我国经济发展来说, 我们只能采取领先发展的策略, 即在提高产品质量、 开发新产品和培养新产业等方面下功夫, 提高经济发展的速度。 